\title{
Validação do World Health Organization Disability Assment Schedule - WHODAS 2.0 em pacientes com Esclerose Múltipla
}

\author{
Validation of the World Health Organization Disability Assment \\ Schedule - WHODAS 2.0 in patients with Multiple Sclerosis
}

\author{
Fabrizio Antonio Gomide Cardoso ${ }^{1}$ \\ Jéssica Mariana de Aquino Miranda ${ }^{1}$ \\ Ana Paula Espindula ${ }^{1}$ \\ Rodrigo César Rosa ${ }^{1}$ \\ Camila Ferreira Leite ${ }^{1}$ \\ Shamyr Sulyvan Castro ${ }^{1}$
}

\section{RESUMO}

O World Health Organization Assessment Schedule(WHODAS 2.0) é um instrumento de avaliaçãogenérico, para avaliação de funcionalidade tendo como referência àClassificação Internacional de Funcionalidade, Incapacidade e Saúde (CIF), a versão brasileira foi traduzida e disponibilizada para uso pela OMS em 2010. 0 objetivo do presente estudo foi validar a versão brasileira do WHODAS 2.0 para pessoas com Esclerose Múltipla (EM). Participaram do estudo 34 indivíduos diagnosticados com EMcom idade média de 41,14 $( \pm 13,31)$ anos e Expanded Disability Status Scale(EDSS) médio de 2,95 ( $\pm 2,62)$. Foram utilizados três instrumentos de avaliação, o WHODAS 2.0 na versão de 36 itens, World Health Organization Quality of Life(WHOQoL-bref) e a EDSS. As avaliações foram realizadas por profissionais capacitados e com expertise na área.As propriedades psicométricas testadas foram consistência interna e validade convergente (testes de correlação de Spearman e Pearson).Este estudo apresentou de forte á moderado índice do coeficiente "alfa de Cronbach" $(0,89)$ e uma correlação forte na maioria dos domínios da EDSS e do WHOQolbref, exceto no domínio relações interpessoais. Conclui-se que o instrumento WHODAS 2.0 é válido para avaliação da funcionalidade de pessoas com EM.As propriedades psicométricas desse questionário apresentam uma validade convergente e consistência interna adequadas para a avaliação funcional de pessoas com EM e pode ser considerada um instrumento útil para medir o suporte funcional desses indivíduos, possibilitando a identificação das áreas mais vulneráveis e as que necessitam de intervenções adicionais.

\section{PALAVRAS-CHAVES}

Esclerose múltipla; Estudos de validação; Psicometria.

\footnotetext{
${ }^{1}$ Universidade Federal do Triângulo Mineiro
} 


\section{ABSTRACT}

The World Health Organization Assessment Schedule (WHODAS 2.0) is a generic assessment tool, created by WHO to assess functionality with reference to the International Classification of Functionality, Disability and Health (CIF), the Brazilian version was translated and made available for use by WHO in 2010. The aim of the present study was to validate the Brazilian version of WHODAS 2.0 for people with Multiple Sclerosis (EM). The study included 34 individuals diagnosed with EM with a mean age of 41.14 ( \pm 13.31 ) years and an Expanded Disability Status Scale (EDSS) average of $2.95( \pm 2.62)$. Three assessment instruments were used, the WHODAS 2.0 in the 36-item version, the World Health Organization Quality of Life (WHOQoL-bref) and the EDSS. The evaluations were carried out by trained professionals with expertise in the area.The psychometric properties tested were internal consistency and convergent validity (Spearman and Pearson correlation tests). This study presented a strong to moderate index of the "Cronbach's alpha" coefficient (0.89) and a strong correlation in most domains of EDSS and WHOQol-bref, except in the interpersonal relations domain. It is concluded that the WHODAS 2.0 instrument is valid for assessing the functionality of people with EM.The psychometric properties of this questionnaire have a convergent validity and adequate internal consistency for the functional assessment of people with EM and can be considered a useful instrument to measure the functional support of these individuals, enabling the identification of the most vulnerable areas and those that need additional interventions.

\section{KEYWORDS}

Multiple sclerosis; Validation studies; Psychometrics. 


\section{INTRODUÇÃO}

Esclerose Múltipla (EM) é definida como uma doença inflamatória, desmielinizante, progressiva, crônica e autoimune que afeta primordialmente a substância branca do Sistema Nervoso Central (SNC) $)^{1,2,3}$. Acomete cerca de 15/100.000 habitantes no território brasileiro. É classificada como remitente recorrente (EMRR), primariamente progressivo (EMPP), secundariamente progressivo (EMSP), progressivorecorrente (EMPR), maligna e benigna. Dessas amais prevalente é a EMRR, atingindo mais de 70\% dospacientes $^{4,5}$. Inicialmente, o impacto da EM pode ser notado pelos sinais e sintomas apresentados, que são dependentes da área afetada no $\mathrm{SNC}^{3}$, sendo os principais sintomas, aparestesia, os distúrbios visuais (diplopia/visão turva), as disfunções vesicais e/ou intestinais, a espasticidade, a fadiga e os sintomas cerebelares (tremor/ataxia) ${ }^{6}$. Podendo acometer os sistemas piramidal, cerebelar, de tronco cerebral, sensorial, vesical, intestinal, visual e mental, levando a sintomatologias diferentes ${ }^{7,8}$.Além de estruturas e funções do corpo, a EM tem impacto mais amplo na funcionalidade do sujeito ao prejudicar a estabilidade postural $^{9}$; a destreza manual ${ }^{10}$; a preparação de refeições;a escrita manual ${ }^{11}$; a manutenção e a mudança da posição corporal; assim como o ato de tomar banho; de caminhar; de fazer o trabalho doméstico; o relacionamento familiar e íntimo; e a vida econômica, social e espiritual ${ }^{12}$.

Os instrumentos mais utilizados para mensurar a qualidade de vida na EM, são: SF-36, Functional Assessment of Multiple Sclerosis, Multiple Sclerosis Quality of Life-54, Sickness Impact Profile, Disability \& Impact Profile, Multiple Sclerosis Impact Scale e Multiple Sclerosis Quality of Live Inventory. Entretanto, os impactos da EM vão além da sintomatologia, implicando em alterações funcionais. A funcionalidade, por sua vez é definida como um conceito que envolve a integração de todos os sistemas corporais, atividades e participação ${ }^{13}$. Percebe-se dessa forma, que profissionais clínicos e pesquisadores da área da saúde devem compreender os impactos daEMparaalémdasintomatologiaapresentada, se preocuparem também com a funcionalidade dos pacientes.A Organização Mundial de Saúde (OMS), atenta à questão da funcionalidade humana, lançou a
Classificação Internacional de Funcionalidade, Incapacidade e Saúde (CIF) que propõe a compreensão da funcionalidade como fruto da interação entre condições de saúde, função e estruturas do corpo, atividades,participaçãoefatorescontextuais(ambientais e pessoais $)^{13}$.Com o intuito de oferecer opções no processo de aplicação da CIF, a OMS publicou o World Health Organization Disability Assessment Schedule(WHODAS 2.0),é um instrumento prático e genérico de avaliação de saúde e deficiência no âmbito populacional ou clínico em diferentes populações ${ }^{14}$, a versão brasileira foi traduzida e disponibilizada para uso pela OMS em 2010.0 instrumento é apresentado em três versões: uma completa com 36 itens, outra resumida com 12, e uma terceira combinando $12+24$ itens. A versão completa, tem sido a mais estudada e utilizada para as propriedades de invariância e psicométricas. A versão combinada é um híbrido das versões completa e resumida. A partir da resposta positiva aos 12 itens iniciais, são aplicadas mais 24 questões. E a versão resumida é indicada em situações em que o tempo não permite uma avaliação mais detalhada e para o uso em estudos populacionais ${ }^{15,16}$.

Naliteratura pesquisada foi encontrado um estudo de revisão de artigos de validação da WHODAS 2.0, de 2009 a 2015, encontrou um interesse no estudo das propriedades psicométricas do instrumento, com 17 artigos de validação em 16 países. Os estudos avaliados, concluíram que o instrumento é uma escala válida e confiávelparaaavaliaçãodaincapacidadeefuncionalidade em diferentes grupos populacionais, incluindo pessoas com diferentes condições de saúde e também indivíduos saudáveis ${ }^{17}$.Assim reforçamos a importância de sua utilização nas redes de atenção a saúde.

O WHODAS 2.0 fornece o nível de funcionalidade de seis domínios de vida:1. Cognição, compreensão e comunicação; 2. Mobilidade, movimentação e locomoção; 3. Autocuidado, lidar com a própria higiene, vestir-se, comer e permanecer sozinho; 4 . Relações interpessoais, interações com outras pessoas; 5. Atividades de vida, responsabilidades domésticas, lazer, trabalho e escola e; 6. Participação, participar em atividades comunitárias e na sociedade ${ }^{18}$.

Embora já existam na literatura instrumentos usados para avaliação de pessoas com EM, como a Expanded Disability Status Scale (EDSS) ${ }^{8}$, eles foram 
criados previamente à CIF, portanto não carregam a concepção de funcionalidade preconizada pela OMS.

A validação deste instrumento genérico que avalia funcionalidade segundo o modelo proposto pela CIF poderá trazer benefícios diversos à pacientes, profissionais e pesquisadores da área da saúde e sistemas de saúde.Os pacientes serão beneficiados pela possibilidade de acesso à tratamento amplo e abrangente, compreendendo não somente a sintomatologia mas também os impactos funcionais da doença; profissionais de saúde terão à disposição ferramenta que quantifica afuncionalidade dos pacientes, podendo servir para orientação e acompanhamento das intervenções propostas; pesquisadores da área da saúde também poderão utilizar uma ferramenta de estudo da funcionalidae entre pessoas com EM com a mesma abordagem da CIF; o sistema de saúde poderá ter informações sobre a descrição numérica do perfil funcional de pacientes com EM.Portanto, o objetivo deste estudo foi validar a versão brasileira do WHODAS 2.0para uso no processo de aferição da funcionalidade entre pessoas com EM.

\section{METODOLOGIA}

0 presente estudo adotou um desenho observacional, descritivo e quantitativo. 0 estudo foi aprovado pelo Comitê de Ética em Pesquisa da Universidade Federal do Triângulo Mineiro (UFTM), sob o protocolo 948.994. Todos os participantes assinaram o termo de consentimento livre e esclarecido (TCLE).

Participaram do estudo 34 indivíduos com EM atendidos no setor de neurologia do Hospital de Clínicas (HC-UFTM), residentes da macrorregião de Uberaba por demanda espontânea. O processo de seleção ocorreu por conveniência, ao convidar os pacientes em acompanhamento para a participação desta pesquisa. Como critério de inclusão cita-se: indivíduos diagnosticados com EM cadastrados no Ambulatório Central Maria da Glória da UFTM e foram excluídos os sujeitos com doenças crônicas associada à EM.

\section{PROCEDIMENTOS}

Uma equipe de entrevistadores treinados para a aplicação do WHODAS 2.0 e com expertise na área, realizou a coleta de dados por um período de 11 meses, administrando o instrumento e entrevistando os participante sem um local reservado, antes ou após a consulta de acompanhamento rotineira no ambulatório de neurologia, o tempo médio de coleta foi de 22 minutos, com cinco minutos para mais ou menos.

\section{INSTRUMENTOS}

Para este estudo foram utilizados os instrumentos: World Health Organization Disability Assessment Schedule - WHODAS 2.0; o World Health Organization Qualityof Life - WHOQoL- Bref; e aExpanded Disability Status Scale(EDSS).

O WHODAS 2.0 para a aferição da funcionalidade ao redor do mundo, já apresenta versão traduzida para uso no Brasil ${ }^{19}$. Existemcinco possibilidades de resposta para cada questão, em uma escala do tipo "likert" que varia de 0 -Nenhuma dificuldade até dificuldade 5-Extrema ou não consegue fazer, gerando escores que varia de zero a 100 para cada domínio e ainda outro escore geral. Quanto maior o escore, pior é a funcionalidade ${ }^{18}$. O WHODAS tem sido validado e usado em países do mundo inteiro para aferição da funcionalidade em sujeitos com perfis de saúde diversos como doenças crônicas ${ }^{20}$, osteoartrite ${ }^{21}$, doença de Huntington $^{22}$, esquizofrenia ${ }^{23}$ e até mesmo entre sujeitos com EM ${ }^{24}$

O WHOQoL-Bref uma versão abreviada do World Health OrganizationQualityof Life - WHOQoL-100, traduzido e validado para uso no Brasil ${ }^{25}$. Este também é um instrumento recomendado pela OMS e possui questões que são divididas e pontuadas segundo quatro domínios: Físico, Psicológico, Social, e Ambiental ${ }^{26}$. As respostas de cada questão tem padrão de escala "likert", variando de um a cinco, produzindo escores por domínio, assim como um escore geral ${ }^{27}$. Os escores variam de zero a 100 , onde 100 seria a melhor qualidade devida ${ }^{28}$.

A Expanded Disability Status Scale traduzida como Escala de Incapacidade Funcional Expandida (EDSS) avalia o grau de incapacidade de pacientes com EM. Esta é dividida emsistemas funcionais, sendo: Piramidal, Cerebelar, Tronco cerebral, Sensorial, Vesicale Intestinal, Visual, Mental e outros. Cada sistema funcional é independe, porém ligados formam o comprometimento neurológico do indivíduo ${ }^{8}$. Estes sistemas avaliam e 
classificam a EM em escores que pode variar de zero a 10 , sendo 10 a morte por EM e zero, nenhuma incapacidade ${ }^{29}$.Porémestaescalapriorizaa deambulação e não é tão sensível aos outros sintomas ${ }^{30}$.

\section{ANÁLISE ESTATÍSTICA}

A consistência interna foi estudada pelo cálculo do coeficiente $\alpha \alpha$ de Cronbach. A validade convergente foi verificadapelos testes de correlaçãodeSpearman(EDSS) e Pearson(WHOQoL) entre os domínios desses instrumentos com os domínios do WHODAS 2.0. A Tabela 1 mostra as suposições sobre correlações convergentes e divergentes estabelecidas a priori. 0 nível de significância adotado foi de $5 \%$. Todos os dados foram tabulados em planilhas e analisados usando-se o programa Stata 13.2.

Tabela 1. Distribuição dos coeficientes de correlação entre os domínios dos instrumentos estudados. C: Convergentes.

\begin{tabular}{|c|c|c|c|c|c|c|c|}
\hline \multirow[t]{2}{*}{ Instrumento/Domínio } & \multicolumn{7}{|c|}{ WHODAS 2.0/Domínios } \\
\hline & Cognição & Mobilidade & $\begin{array}{l}\text { Auto- } \\
\text { cuidado }\end{array}$ & $\begin{array}{l}\text { Relações } \\
\text { interpessoais }\end{array}$ & $\begin{array}{l}\text { Atividades } \\
\text { de vida }\end{array}$ & Participação & Total \\
\hline \multicolumn{8}{|l|}{ WHOQoL } \\
\hline Domínio Físico & & $\mathrm{C}$ & $\mathrm{C}$ & & $\mathrm{C}$ & $\mathrm{C}$ & $\mathrm{C}$ \\
\hline Domínio Pscicológico & $\mathrm{C}$ & & & & $\mathrm{C}$ & $\mathrm{C}$ & $\mathrm{C}$ \\
\hline Domínio Social & $\mathrm{C}$ & & & $\mathrm{C}$ & $\mathrm{C}$ & $\mathrm{C}$ & $\mathrm{C}$ \\
\hline Domínio Ambiental & $\mathrm{C}$ & & $\mathrm{C}$ & & & $\mathrm{C}$ & $\mathrm{C}$ \\
\hline Total & $\mathrm{C}$ & $\mathrm{C}$ & $\mathrm{C}$ & $\mathrm{C}$ & $\mathrm{C}$ & $\mathrm{C}$ & $\mathrm{C}$ \\
\hline \multicolumn{8}{|l|}{ EDSS } \\
\hline Piramidais & & $\mathrm{C}$ & $\mathrm{C}$ & & $\mathrm{C}$ & $\mathrm{C}$ & $\mathrm{C}$ \\
\hline Cerebelares & & $\mathrm{C}$ & $\mathrm{C}$ & & $\mathrm{C}$ & & $\mathrm{C}$ \\
\hline Tronco cerebral & & $\mathrm{C}$ & & & & & \\
\hline Sensitivas & & $\mathrm{C}$ & $\mathrm{C}$ & & $\mathrm{C}$ & & $\mathrm{C}$ \\
\hline Vesicais & & $\mathrm{C}$ & $\mathrm{C}$ & & $\mathrm{C}$ & & $\mathrm{C}$ \\
\hline Intestinais & & $\mathrm{C}$ & & & & & \\
\hline \multicolumn{8}{|l|}{ Visuais } \\
\hline M Mentais & & $\mathrm{C}$ & & & & & $\mathrm{C}$ \\
\hline Outras & & $\mathrm{C}$ & & & $\mathrm{C}$ & & \\
\hline Total & & $\mathrm{C}$ & $\mathrm{C}$ & & C & $\mathrm{C}$ & $\mathrm{C}$ \\
\hline
\end{tabular}

\section{RESULTADOS}

Participaram do estudo 34 indivíduos, cadastrados no local do estudo,com idade média 41,14 anos (DP: 13,31), sendo (61,76\%) do gênero feminino,estado civil casados (50\%), e 56\% da amostra estavam em condição de aposentadoria, escolaridade de 12,79 anos de estudos (DP: 7,31). Os indivíduos apresentavamem sua maioria a classificação da doença EMRR (77\%), EDSS com média de 2,95 e média de diagnóstico 9,01 meses (DP: 5,40)(Tabela 2). 
Tabela 2. Caracterização da amostra.

\begin{tabular}{lll}
\hline Variáveis & $\mathrm{n}(34)$ & $\%(100,00)$ \\
& & \\
\hline Gênero & 13 & 38,24 \\
$\quad$ Masculino & 21 & 61.76 \\
$\quad$ Feminino & & \\
Estado civil & 11 & 32,36 \\
$\quad$ Nunca se casou & 17 & 50,00 \\
$\quad$ Atualmente casado(a) & 1 & 2,94 \\
Separado(a) & 1 & 2,94 \\
Divorciado(a) & 3 & 8,82 \\
Viúvo(a) & 1 & 2,94 \\
Mora junto & & \\
Situação de trabalho & 19 & 55,88 \\
$\quad$ Aposentado & 1 & 2,94 \\
$\quad$ Estudante & 14 & 41,18 \\
$\quad$ Outro & & \\
Classificação da Esclerose Múltipla & 26 & 76,47 \\
$\quad$ Remitente recorrente & 2 & 5,88 \\
Primariamente progressiva & 6 & 17,65 \\
Secundariamente progressiva & Média & Desvio-padrão \\
& 41,14 & 13,31 \\
Idade (anos) & 2,95 & 2,62 \\
EDSS & 12,79 & 7,31 \\
Anos de estudo & 9,06 & 5,40 \\
Tempo de diagnóstico (meses) ${ }^{1}$ & & \\
\hline
\end{tabular}

Dados não encontrados na descrição dos prontuários de cinco sujeitos nessa variável.

A consistência interna foi testada a partir do coeficiente $\alpha \alpha$ de Cronbach e obtiveram-se coeficientes de moderado a forte, para os domínios: Auto-cuidado $(0,73)$, Cognição $(0,77)$, Mobilidade $(0,85)$, Participação $(0,85)$ e Atividades de vida $(0,88)$, e fraco para relações Interpessoais $(0,58)$, tendo como coeficiente $\alpha \alpha$ de Cronbach total $(0,89)$.

Os coeficientes entre WHODAS 2.0 e WHOQOL-Bref apresentaram correlação forte e negativa, principalmente no domínio Físico $(-0,75)$ e domínio do Meio ambiente $(-0,75)$. Já WHODAS 2.0 com EDSS à correlação positiva moderada principalmente nas funções piramidais $(0,63)$ e nas funções cerebelares $(0,54)$ (Tabela 3).

\section{DISCUSSÃO}

O presente estudo objetivou validar a versão brasileira do WHODAS 2.0 para uso no processo de aferição da funcionalidade entre pessoas com EM. E apresentou perspectiva psicométricas significantes para a sua validação nessa população. 0 alpha Cronbach teve índice de forte á moderado e também correlações para Whoqol-Bref e EDSS. Magistrale, assim como este estudo, encontrou fortes características psicométricas para pacientes com $\mathrm{EM}^{24}$.

Os coeficientes alpha Cronbach se manifestaram acima de 0,70 na maioria dos domínios, o que qualifica o desempenho do instrumento, segundo a literatura pesquisada, como bom ${ }^{31}$, com exceção do domínio 
Relações Interpessoais. Este índice se apresentou um pouco inferior em validações realizadas com doenças crônicas $^{20}, \mathrm{EM}^{24}$ e Acidente Vascular Encefálico ${ }^{32}$, pois nestes estudos o índice total foi maior que 0,90. Porém apresenta um número significante para validação. Alguns estudos já encontraram coeficientes baixos no domínio Relações Interpessoais ${ }^{20,24,33}$ e a argumentação usada é a de que as duas questões sobre relações sexuais e relações com pessoas próximas a você seriam perguntas relacionadas à tabus sociais e introduziriam variação na coleta dos dados por conta de percepções e valores subjetivos.

A validade externa foi testada pela comparação do WHODAS 2.0 com o WHOQoL-Bref e com a EDSS. A comparação entre os domínios de WHODAS 2.0 e WHOQoL-Bref mostrou correlações significantes entre o domínio cognição do WHODAS com os domínios Psicológico, Social e Ambiental do WHOQoL-Bref.O domínio Mobilidade do WHODAS 2.0 teve correlação com os domínios Físico e Psicológico do WHOQoL-Bref; Auto-cuidado do WHODAS 2.0 mostrou correlação com os domínios Físico e Ambiental do WHOQoL-Bref; o domínio Relações Interpessoais do WHODAS 2.0 teve correlação com o domínio Social do WHOQoL-Bref; Atividades de vida do WHODAS mostrou correlação com os domínios Físico, Psicológico e Social do WHOQoLBref; e o domínio Participação do WHODAS 2.0 teve correlação com todos os domínios do WHOQoL-Bref. Verificou-se forte correlação entre domínio Físico do WHOQoL-Bref com o domínio Mobilidade, Atividades de vida e participação do WHODAS 2.0 e também entre o domínio Social com relações interpessoais. Outros autores também registraram de moderada a forte correlação negativa para estes itens, realçando a ligação entre WHODAS 2.0e questionários de qualidade de vida, incluindo a escala WHOQoL-Bref ${ }^{24,32}$.

A escala EDSS utilizada neste estudo tem uma base diferente do WHODAS 2.0e do WHOQol-Bref, ela visa classificar as incapacidades do indivíduo por meio de sistemas funcionais diferentes e independentes, todavia quando se reúnem formam um organismo unificado que apresenta as incapacidades presentes ${ }^{8}$. Quando se correlaciona WHODAS 2.0com a EDSS podemos identificar o quanto duas escalas com abordagens diferentes de funcionalidade e/ou incapacidade podem se relacionar. O domínio Mobilidade se correlaciona com todos os sistemas da EDSS, exceto o sistema Visual. Ao passo que, os domínios Auto cuidado e Atividade de vida do WHODAS 2.0 se correlacionam fortemente com os sistemas Piramidal, Cerebelar, Sensitivo e Vesical. No entanto as medidas Psicométricas entre os domínios Cognição, Relações Interpessoais e Participação não se correlacionam fortemente com nenhum sistema específico apresentados pela EDSS.Nos resultados encontrados, depara-se com uma EDSS com score com média de 2,95,indicando que osindivíduos apresentam, em sua maioria, uma incapacidade moderada em um sistema funcional ou uma incapacidade discreta em três ou quatro sistemas funcionais, no entanto deambulam plenamente ${ }^{8}$.Com isso, os resultados encontrados denotam uma correlação forte entre os domínios Mobilidade $(0,81)$ e Atividades de vida(0,69) com a EDSS. De acordo com o descrito na literatura que observou que a escala teve uma correlação maior nas atividades que priorizam a deambulação ${ }^{29}$.Um dos principais problemas da EDSS é justamente o seu direcionamento dado à deambulação, e questões muito gerais relacionadas aos sistemas funcionais, não levantando questões cognitiva e social do paciente ${ }^{34}$ sendo essa uma questão muito bem abordada no WHODAS 2.0 e seus domínios, justificando a importância da validação.

Nossos resultados convergem com os apresentados por outros estudos realizados em indivíduos com outras disfunções que tem como característica a cronicidade e a progressividade, assim como a EM. Tais como:Osteoartrite ${ }^{21}$, doenças crônicas ${ }^{20}$ e Artrite Reumatoide ${ }^{21}$, que apresentaram resultados significativos para a validação do WHODAS 2.0. Sendo assim,evidenciam correlações, índice de alpha Cronbachalém de outras análises estatísticas de forte significância que respaldama validação deste questionário. Com isso, os resultados aqui apresentados condizem com a validação do instrumento.Este estudo, é a primeira validação para indivíduos com EM no Brasil. Entretanto, nota-se que apresentou baixa amostrageme aplicação de dois testes psicométricos. Porém, apresentou resultados significantes, o que demonstrou ser eficaz para esta população. 


\section{CONCLUSÃO}

O nível de funcionalidade de cognição, mobilidade, autocuidado, relações interpessoais, atividades de vida e participação em atividades comunitárias e na sociedade, desempenha um papel importante na saúde e no bem-estar de indivíduos com EM. Este é o primeiro estudo que visou validar a WHODAS 2.0 em pessoas com EM. As propriedades psicométricas desse questionário apresentam uma validade convergente e consistência interna adequadas para a avaliação funcional de pessoas com EM e pode ser considerada um instrumento útil para medir o suporte funcional desses indivíduos, possibilitando aidentificação das áreas mais vulneráveis e as que necessitam de intervenções adicionais, assim como a elaboração de plano de cuidados e planejamento assistencial, com base nos resultados individuais de cada paciente submetido a avaliação da WHODAS 2.0. A identificação dessas propriedades permite que os profissionais da reabilitação intervenham diretamente como um recurso valioso de suporte para a promoção do cuidado, do autocuidado e do bem-estar.Osresultados provavelmente contribuirão para a educação e formação de profissionais e estudantes. Além disso, o uso da WHODAS 2.0 pode ser amplamente estendido aos contextos de aplicabilidades nos indivíduos com EM para apoiar futuras pesquisas. 


\section{REFERÊNCIAS}

BARON, M.; SCHIEIR, O; HUDSON, M. et al. The Clinimetric Properties of the World $\mathrm{Health}$ Organization Disability Assessment Schedule II in Early Inflammatory Arthritis. Arthritis \& Rheumatism 2008;59:382-390.

BROCHET, B. Place de l'EDSS dans l'évaluation précoce du handicap: Assessing incapacity at early stages of Multiple sclerosis using the EDSS. Revue Neurologique 2009;165:173179.

CARLOZZI, N. E.; KRATZ, A. L.; DOWNING, N.R.et al. Validity of the 12-item World Health Organization Disability Assessment Schedule 2.0 (WHODAS 2.0) in individuals with Huntington disease (HD). Quality of Life Research 2015; 24:1963-1971.

CASTRO, S. S.; LEITE, C.F.; OSTERBROCK, C.; SANTOS, M.T.; ADERY, R. Avaliação de Saúde e Deficiência: Manual do WHO Disability Assessment Schedule (WHODAS 2.0). Uberaba: Universidade Federal do Triângulo Mineiro - UFTM, 2015.

Centro Colaborador da Organização Mundial da Saúde para a Família de Classificações Internacionais, organizador. CIF: Classificação Internacional de Funcionalidade, Incapacidade e Saúde. São Paulo: Editora da Universidade de São Paulo; 2003.

CHIU, T. Y.; YEN, C. F; CHOU, G. H. et al. Development of traditional Chinese version of World Health Organization Disability Assessment Schedule 2.036 - item (WHODAS 2.0) in Taiwan: Validity and reliability analyses. Research in Developmental Disabilities 2014;35:2812-2820.

COENEN, Michaela; CIEZA, Alarcos; FREEMAN, Jennyet al. The development of ICF core sets for multiple sclerosis: results of the international consensus conference. J. Neurol. 2011;258:1477-1488.

COSTA, R. M. A. Qualidade de vida na esclerose múltipla: casuística do Centro Hospitalar Cova da Beira. 48f. Dissertação (Mestrado em medicina - ciclo de estudos integrado). 2013.

Federici S, Bracalenti M, Meloni F, Luciano JV. World Health Organization Disability Assessment Schedule 2.0: an international systematic review. Disabil Rehabil. 2017;39(23):2347-80.

FELIPE, E.; MENDES, M. F.; MOREIRA, M.A.; TILBERY, C.P. Análise comparativa entre duas escalas de avaliação clínica na esclerose múltipla: revisão de 302 casos. Arquivos de NeuroPsiquiatria 2000;58:300-303.

FLECK, M. P.; LOUZADA, S; XAVIER, M. et al.Aplicação da versão em português do instrumento abreviado de avaliação da qualidade de vida "WHOQOL-bref". Revista de Saúde Pública 2000;34:178-183.
GARIN, O; MATEOS, J. L. A.; ALMANSA, M.et al.Validation of the "World Health Organization Disability Assessment Schedule, WHODAS-2" in patients with chronic diseases. Health and quality of life outcomes 2010;8:1-15.

Gaskin CJ, Lambert SD, Bowe SJ, Orellana L. Why sample selection matters in exploratory factor analysis: implications for the 12-item World Health Organization Disability Assessment Schedule 2.0. BMC Med Res Methodol. 2017;17(1):40.

GHOLAMI, A.JAHROMI, L.M.; ZAREI, E.; DEHGHAN,A. Application of WHOQOL-BREF in Measuring Quality of Life in Health-Care Staff. International Journal of Preventive Medicine 2013;4:809-17.

GUILERA, G.; BENITO, J. G.; PINO, O.et al.Utility of the World Health Organization Disability Assessment Schedule II in schizophrenia. Schizophrenia Research 2012;138:240-247.

HOLPER, Lisa; COENEN, Michaela; WEISE, Andrea; STUCKI, Gerold; CIEZA, Alarcos; KESSELRIN, Jürg. Characterization of functioning in multiple sclerosis using the ICF. J. Neurol. 2010;257:103-113.

KÜÇÜKDEVECI, A.A.; KUTLAY, S.; YILDIZLAR, D.; ÖZTUNA, D; ELHAN, A.H.; TENNANT, A. The reliability and validity of the World Health Organization Disability Assessment Schedule ( WHODAS-II) in stroke. Disability \& Rehabilitation 2013;35:214-220.

KURTZKE, John F. Rating neurologic impairment in multiple sclerosis: an expanded disability status scale (EDSS). Neurology 1983;33:1444-1452.

KURTZKE, John F.; BEEBE, Gilbert W.; NAGLER, Benedict; AUTH, Thomas L.; KURLAND, Leonard T.; NEFZGER, M. Dean. Studies on the history of multiple sclerosis. Acta. Neurol. Scandinav 1972;48:19-46.

KUTLAY, S; KÜÇÜKDEVECI, A. A.; ELHAN, A. H.; ÖZTUNA, D.; KOÇ, N.; TENNANT, A. Validation of the World Health Organization disability assessment schedule II (WHODAS-II) in patients with osteoarthritis. Rheumatology international 2011;31:339-346.

LOPES, K.N; NOGUEIRA, L.A.C; NÓBREGA, F.R; FILHO, H.A; ALVARENGA R.M.P. Limitação funcional, fadiga e qualidade de vida na forma progressiva primária da esclerose múltipla. Revista Neurociência. 2010;18(1):13-17.

MAGISTRALE, G.; PISANI, V.; ARGENTO, O. et al.Validation of the World Health Organization Disability Assessment Schedule II (WHODAS-II) in patients with multiple sclerosis. Multiple Sclerosis Journal 2015;21: 448-456.

MENDES, M. F.; BALSIMELLI, S.; STANGEHAUS, G; TILBERY, C.P. Validação de escala de determinação funcional da qualidade de 
vida na esclerose múltipla para a língua portuguesa. Arquivos de Neuro Psiquiatria 2004;62:108-113.

MENDES, M.F; TILBERY, C.P; FELIPE, E. Fadiga e Esclerose Múltipla. Estudo de 15 Casos Através de escalas de AutoAvaliação. Arq Neuropsiquiatr. 2000; 58(2-b):467-470.

MENDES, Maria Fernanda; TILBERY, Charles Peter; BALSIMELLI, Silvia; MOREIRA, Marcos Aurélio; CRUZ, Ana Maria Barão. Teste de destreza manual da caixa e blocos em indivíduos normais e em pacientes com escleroses múltipla. Arq. Neuropsiquiatr. 2001; 59:889-894.

MENEZES, Karla Mendonça; COPETTI, Fernando; WIEST, Matheus Joner; TREVISAN, Cláudia Morais; SILVEIRA, Aron Ferreira. Efeito da equoterapia na estabilidade postural de portadores de esclerose múltipla: estudo preliminar. Fisioter. Pesq. 2013;20:43-49.

Ministério da Saúde Secretaria de Atenção à Saúde Secretaria de Ciência, Tecnologia e Insumos Estratégicos Portaria Conjunta № 10, de 02de abril de 2018. Aprova o Protocolo Clínico e Diretrizes Terapêuticas da Esclerose Múltipla. http:// portalarquivos2.saude.gov.br/images/pdf/2018/abril/09/ portaria-conjunta-n-10-esclerose-multipla. 2018.

Organização Mundial da Saúde. Avaliação de saúde e deficiência: manual do WHO Disability Assessment Schedule 9 (WHODAS 2.0). Uberaba: Universidade Federal do Triangulo Mineiro; 2015 [cited 2017 Aug 7].

PIMENTEL, P.P; TOLDRÁ, R.C. Desenvolvimento de manual para orientações básicas do dia a dia para pessoas com esclerose múltipla. Cadernos de Terapia Ocupacional. UFSCar. 2017; 25, n. 1, p. 67-74.

Silveira, L.S. World Health Organization Disability Assessment Schedule (WHODAS 2.0): revisão de literatura dos processos de validação em grupos distintos e aferição das propriedades psicométricas da versão brasileira para cegos. Uberaba: Universidade Federal do Triângulo Mineiro, 2016. Dissertação.

SKEVINGTON, S. M.; LOTFY, M.; O'CONNELL, K.A. The World Health Organization's WHOQOL-BREF quality of life assessment: psychometric properties and results of the international field trial. A report from the WHOQOL group. Quality of life research : an international journal of quality of life aspects of treatment, Care and Rehabilitation 2004;13:299-310.

THE WHOQOL GROUP. The World Health Organization Quality of Life assessment (WHOQOL): position paper from the World Health Organization. Social science \& medicine 1995;41:1403-1409.

TORO, G. J; PATIÑO, J; REYES, S.L; RIVERA, J.S; RÍOS, J; NORIEGA, D.Qué se debe saber en esclerosis múltiple: 16 preguntas prácticas / What one needs to know in multiple sclerosis: 16 practical questions. Acta Neurol. Colomb. 2019;35(1):40-49.

ÜSTÜN, T.; KOSTANJSEK, N; CHATTERJI, S; REHM, J. Measuring health and disability: manual for WHO Disability Assessment Schedule (WHODAS 2.0). Geneva: World Health Organization, 2010 